\title{
Combining Atom-Probe Tomography and Focused-Ion Beam Microscopy to Study Individual Presolar Meteoritic Nanodiamond Particles
}

Dieter Isheim $^{1,2}$, Frank J. Stadermann ${ }^{3,4}$, Josiah B. Lewis ${ }^{3,4}$, Christine Floss ${ }^{3,4}$, Tyrone L. Daulton ${ }^{3,4}$ Andrew M. Davis ${ }^{5,6,8}$, Philipp R. Heck ${ }^{6,8}$, Michael J. Pellin ${ }^{5,6,7}$, Michael R. Savina, ${ }^{6,7}$ David N. Seidman ${ }^{1,2}$, Thomas Stephan ${ }^{5,6,7,8}$

${ }^{1}$ Department of Materials Science and Engineering, ${ }^{2}$ Northwestern University Center for Atom-Probe Tomography, Evanston, IL 60208-3108, USA, ${ }^{3}$ Laboratory for Space Sciences, ${ }^{4}$ Physics Department, Washington University, St. Louis, MO, USA, ${ }^{5}$ Department of the Geophysical Sciences, ${ }^{6}$ Chicago Center for Cosmochemistry, University of Chicago, Chicago, IL, USA, ${ }^{7}$ Materials Science Division, Argonne National Laboratory, Argonne, IL, USA, ${ }^{8}$ Robert A. Pritzker Center for Meteoritics and Polar Studies, Department of Geology, The Field Museum, Chicago, IL, USA

A method is presented for atom-by-atom analyses of individual nanometer-sized granular diamond particles (nanodiamonds, NDs) by atom-probe tomography (APT). A metal-deposition technique to affix the NDs is combined with the unique processing capabilities of a dual-beam focused-ion beam (FIB) microscope to obtain microtips suitable for APT. NDs extracted from the primitive carbonaceous Allende meteorite first identified by transmission electron microscopy (TEM) [1]. These NDs have been linked to a presolar origin based on isotopic anomalies of Xe and other trace elements, detected by bulk mass spectrometric methods [1,2] of large numbers of NDs, which, however, showed that the bulk ratio of ${ }^{12} \mathrm{C} /{ }^{13} \mathrm{C}$ is close to the solar system ratio, $89[3,4]$. In this correlative study of the same sample material, we apply APT to measure the isotopic carbon composition of individual Allende NDs [5].

Figure 1 illustrates the preparation technique. A $170 \mathrm{~nm}$ thick $\mathrm{Pt}(\mathrm{Al})$ bottom layer is deposited by ionbeam sputtering (South-Bay Technologies IBS/e) onto a polished Ni substrate disk, Fig. 1a. Next, the NDs are deposited by evaporating a droplet of de-ionized water with the NDs held in suspension by ultrasonic vibration of the Ni disk, resulting in a nearly circular deposition band, Fig. 1c, with individual NDs at the perimeter and larger clusters of NDs in the inner regions. The ND layer is then covered with a $170 \mathrm{~nm} \mathrm{Pt}(\mathrm{Al})$ overlayer and a $400 \mathrm{~nm} \mathrm{Ni}$ cover layer to provide a symmetric geometry for microtip preparation. The ND deposition band can be identified by SEM through the over- and cover layers, Fig. 1c. A cross-sectional cut, Fig. 1b, is made with a FEI Helios Nanolab FIB to locate the zone with individual NDs. A bar-shaped section is lifted-out in the FIB utilizing a standard technique [6], Fig. 1d, with an Omniprobe 200 micromanipulator, and transferred to a rotatable needle, Fig. 1e, for vertical reorientation. Sections of the rotated lift-out bar are mounted on standard silicon microposts [6], Fig. 1f. Employing annular milling patterns, a tip is formed centered on the deposition band within the region containing individual NDs in the microtip's apex, Figs. 1g,h. A Cameca LEAP4000XSi is used for APT analysis. Field-evaporation is assisted by focused ultraviolet $(355 \mathrm{~nm})$ laser pulses with an energy of 40$100 \mathrm{pJ}$ per pulse, and a $500 \mathrm{kHz}$ pulse repetition rate, at a base specimen temperature of $95 \mathrm{~K}$.

Figure 2 is a 3D atom-by-atom APT reconstruction of about 25 individual NDs embedded in the Pt(Al) deposition layer. Figure 3 is the mass spectrum of the NDs, displaying the region of the peaks for ${ }^{13} \mathrm{C}$ and ${ }^{12} \mathrm{C}$, singly and doubly charged ions. A ratio of ${ }^{12} \mathrm{C} /{ }^{13} \mathrm{C}$ of $63 \pm 7$ is obtained for $\mathrm{C}^{+}$, and $78 \pm 10$ for $\mathrm{C}^{++}$. These values are close to the solar system ratio, 89, with differences attributed to the instrumental mass fractionation. 
References

[1] R.S. Lewis et al., Nature 326 (1987), p. 160.

[2] R.S. Lewis et al., Science 190 (1975), p. 1251; S. Richter et al., Nature 391 (1998), p. 261.

[3] S.S. Russell et al., Science 254 (1991), p. 1188; Marty et al., Science 322 (2011), p.1533.

[4] Z.R. Dai et al., Nature 418 (2002), p. 157.

[5] F.J. Stadermann et al., Lunar and Planetary Science Conference XLII (2011) \#1595; P.R. Heck et al., Lunar and Planetary Science Conference XLII (2011) \#2070.

[6] K. Thompson et al., Ultramicroscopy 107 (2007) 131.

[7] This work is supported by NASA grant NNX09AC28G (C.F.). We thank R.S. Lewis for providing the NDs from the Allende meteorite. The LEAP tomograph at NUCAPT was purchased and upgraded with funding from NSF-MRI (DMR-0420532) and ONR-DURIP (N00014-0400798, N00014-0610539, N00014-0910781) grants. Instrumentation at NUCAPT was supported by the Initiative for Sustainability and Energy at Northwestern. This research made use of the EPIC facility and Shared Facilities at the Materials Research Center, supported by the NSF's MRSEC program (DMR-0520513 and 1121262).

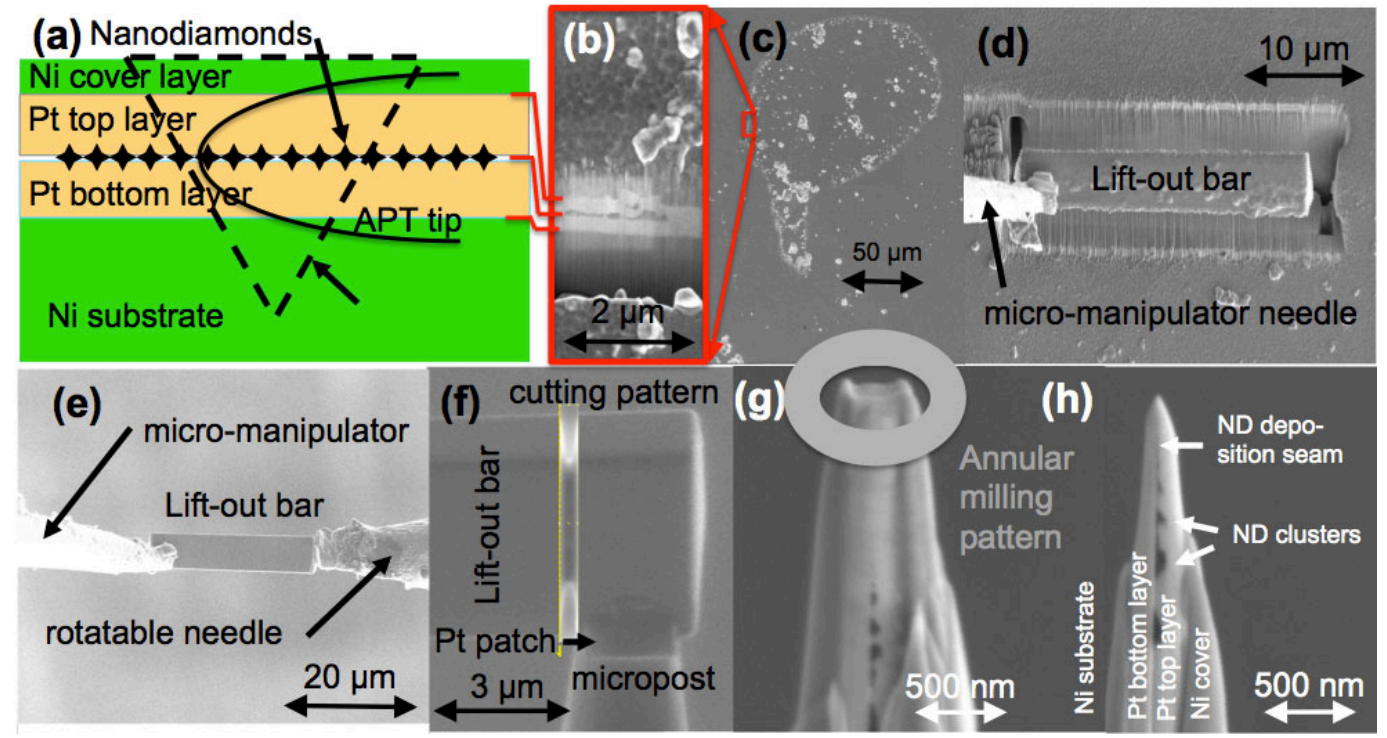

Figure 1. Schematic of deposition layering (a-c) to embed nanodiamonds in a Pt matrix. FIB lift-out (d); lift-out reorientation (e); tip mounting (f); tip sharpening (g); and the final microtip for APT (h).

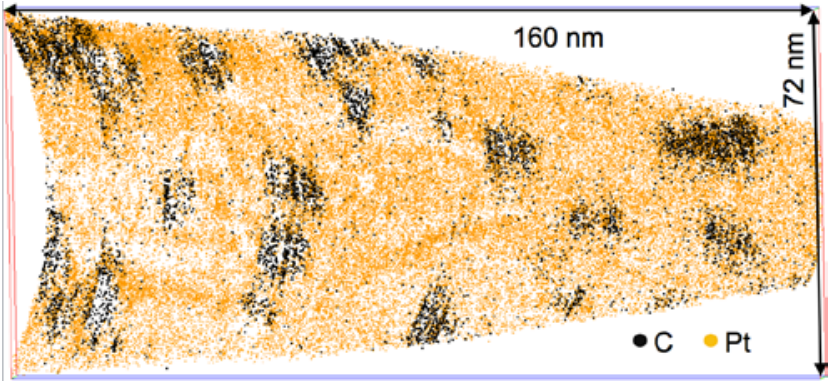

Figure 2. Atom-by-atom 3D reconstruction of individual nanodiamonds extracted from the Allende meteorite, for APT analysis embedded in a $\mathrm{Pt}(\mathrm{Al})$ matrix. $\mathrm{C}$ atoms are represented as black dots, and $\mathrm{Pt}$ atoms in orange. Only 5\% of Pt atoms displayed for clarity.

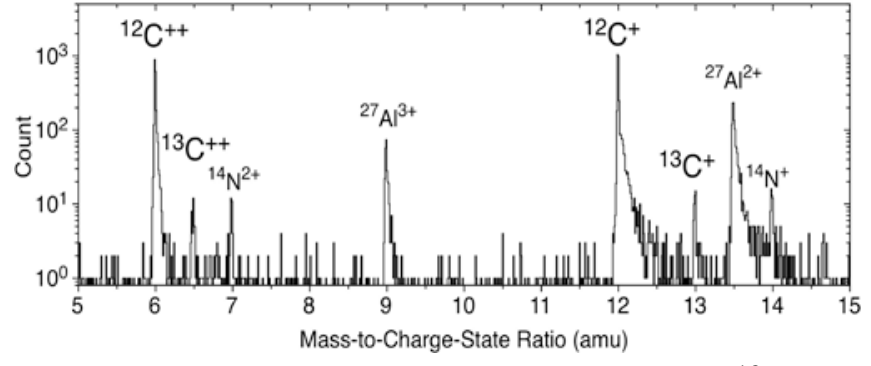

Figure 3. A partial mass spectrum with ${ }^{12} \mathrm{C}$ and ${ }^{13} \mathrm{C}$ isotopic peaks from nanodiamonds extracted from the Allende meteorite. A 5 at.\% carbon isoconcentration surface was used to delineate the NDs in the reconstruction, Fig. 2. The Al peaks are from the $\mathrm{Pt}(\mathrm{Al})$ embedding material. 